\title{
数据操作系统的思想、设计和原型验证
}

\author{
李明树 ${ }^{(2)}$ ，赵琛 ${ }^{(1)}$ ，武延军 ${ }^{(1) *}$, 谢沛东 ${ }^{(1)}$ \\ (1) 中国科学院软件研究所, 北京 100190 \\ (2) 计算机科学国家重点实验室, 北京 100190 \\ * 通信作者. E-mail: yanjun@iscas.ac.cn
}

收稿日期: 2014-11-20；接受日期: 2015-04-06; 网络出版日期: 2015-05-27

中国科学院战略性先导科技专项 (批准号: XDA06010600) 资助

\begin{abstract}
摘要 现代主流操作系统发展至今, 对设备的依赖性越来越低, 与数据的相关性越来越高, 对数据的 支持将成为新一代操作系统的核心功能目标, 这在海云协同网络计算环境下表现得尤为突出. 本文 分析了现有操作系统面临的挑战和存在的问题, 进而提出数据型操作系统的思想和设计, 引入数据 相关的抽象概念, 在操作系统中增加数据栈 (Data Stack), 将数据的感知、传输、处理、存储和服务等 各个层次纳入内核态, 减少不必要的性能开销, 同时为后续通过数据挖掘实现操作系统自适应的升 级演化奠定了基础. 论文实现了一个包含数据栈的数据操作系统原型 DataOS, 把 Key/Value 数据存 储移植到数据栈框架下, 取得了优于现有系统的实时性和并发性, 初步验证了数据操作系统思想的 可行性和架构设计的有效性.
\end{abstract}

关键词 操作系统 数据操作系统 数据栈 大数据 分布式系统

\section{1 背景}

\section{1 海云计算环境的挑战和需求}

随着互联网和物联网的高速发展, 人类信息网络正在高速扩展, 未来的信息网络将涵盖传统人类 社会网络和物理世界的绝大部分信息, 形成集人、机、物于一体的三元融合型信息世界. 以物联网为代 表的信息网络技术促进了信息化终端规模的爆炸式增长, 以云计算模式为核心的信息服务模式将受到 极大的性能挑战. 海计算 [1] 作为中国科学院率先提出的由下一代关键信息技术组成的新型计算模式, 通过强化融入在各种物体中的信息装置, 强化海量独立个体之间的局部即时交互和分布式智能, 使物 体具备自组织、自计算、自反馈的能力.

海云协同的网络计算模式融合了云计算、物联网、大数据、未来网络的诸多特点, 涵盖了泛在感 知设备、移动智能终端、大规模弹性化服务系统、海量云端存储服务等, 是一种人机物融合、海云一 体化、以数据服务为目标的新型计算模式. 它既包含了海终端 (智能设备、感知设备)、未来网络与云 计算的一体化融合, 也覆盖了多种类型数据采集、传输、存储、处理与服务的标准化, 同时要求大数据 服务具有数据弹性化、可管控、可共享、服务智能化, 此外还有计算的多层级局部自治与整体协同的 特性. 


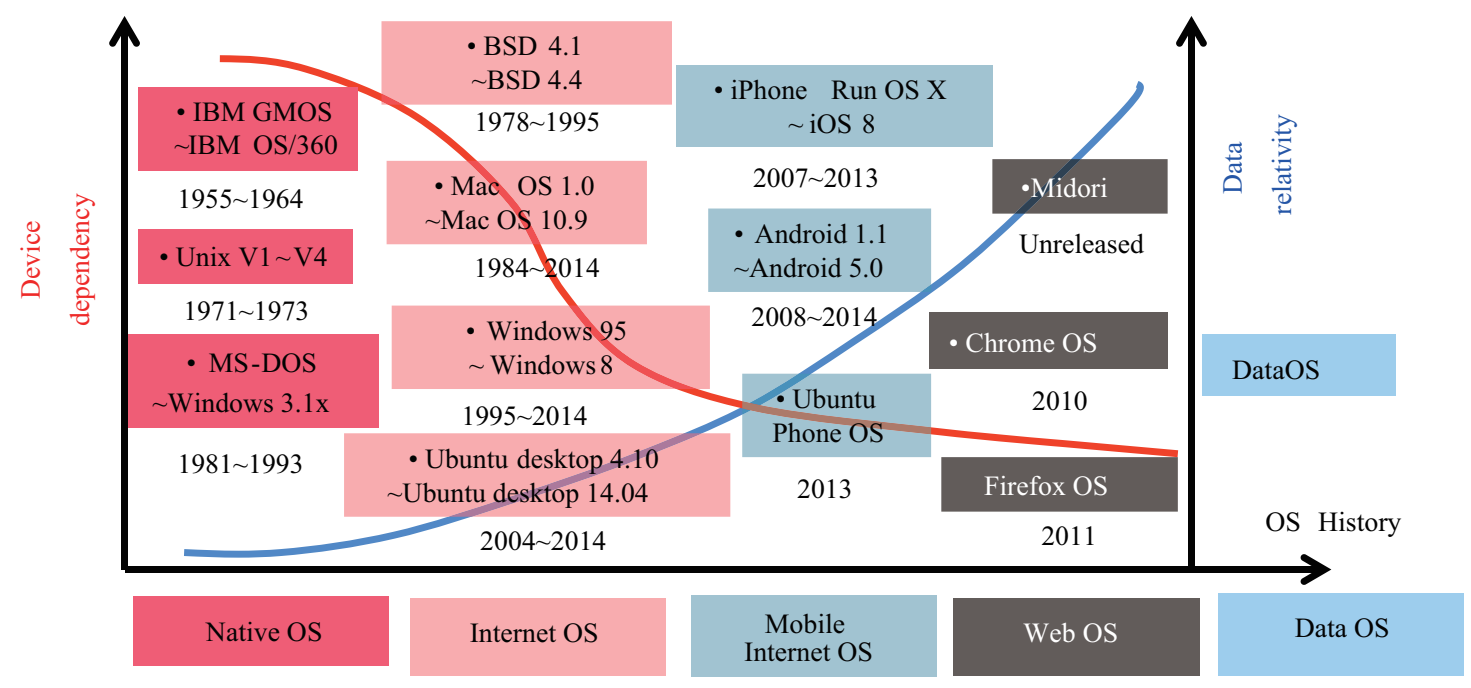

图 1 (网络版彩图) 操作系统基于设备依赖和数据相关的划代

Figure 1 (Color online) OS generations divided by device dependency and data relativity

在海云协同网络计算环境中, 安全、规模、性能、能耗、传输、处理等挑战的解决方案之间存在一 定的矛盾, 如规模与性能、规模与能耗、性能与能耗、性能与安全等因素之间都存在着冲突关系. 但在 给定范围的海云协同网络中, 施加特定约束条件可以找到性能、能耗、安全的平衡点. 这需要网络中 各个节点紧密协同, 充分进行软硬件信息的获取、共享, 而只有操作系统这样的系统级软件才能有效 实现这一目标.

\section{2 操作系统的发展变化}

操作系统的发展伴随着现代计算机发展的历史. 从 20 世纪 $60 、 70$ 年代的大型机到 80 年代开始 的 PC, 从 21 世纪初的移动智能终端到当前的可穿戴设备, 操作系统也经历了一代又一代的发展. 总 体来看, 主导操作系统发展的两大核心因素是设备和数据. 而二者在操作系统发展过程中的影响权重 在发生变化, 即操作系统对设备的依赖性在逐渐下降, 而与数据的相关性在逐渐增加. 由此, 我们可以 把操作系统的历史划分为本地型、网络型、移动互联网型、网页型和数据型 5 个阶段. 这种变化和相 应的划代可以用图 1 来直观表示.

其中, 本地型 OS 的典型代表起始于 20 世纪 60 年代, 以 Multics、IBM 360、早期 Unix 为代表 操作系统, 它们专门为特定的大型主机的硬件系统而研发, 具有很强的硬件专用特性. 网络型 OS 则 是自 BSD 4.1 以后, 具有 TCP/IP 协议栈的各种操作系统, 包括现有的 Window, Linux 等主流操作系 统, 能够运行在多种硬件兼容的设备上, 并可以通过互联网交换信息. 移动互联网型 OS 的典型代表 是 Android 和 iOS, 其重要特点是在网络型 OS 的基础上,在应用和操作系统之间增加了新中间层次 (如 Android 的 Dalvik 虚拟机或 iOS 的媒体层), 使之不再与设备紧密绑定, 数据的移动性进一步加强. 网页型 OS 以新兴的 Chrome OS 和 Firefox OS 为代表, 强调应用的完全 Web 化, 数据随用户账号和 应用按需迁移. 数据型操作系统则是本文所研究的目标操作系统, 是一个能够使应用不再依赖特定设 备、完全以数据为核心的新一代操作系统. 


\section{3 传统操作系统存在的不足}

传统操作系统主要为高效便捷地使用硬件资源而设计, 当数据成为计算的核心时, 这种设计面临 新的挑战, 不能很好地满足计算环境的需求, 具体表现在以下 “不擅长”、“不作为” 和 “不能够”几个 方面.

\subsection{1 传统操作系统的 “不擅长”}

首先, 传统操作系统不擅长数据密集型计算. 事实表明, 把数据从它们的存储区域移动到 CPU 再 移走的时间远远超过了它们在 CPU 停留的时间, 使得数据传输取代 CPU 运算能力成为计算的新瓶 颈. 计算从 $\mathrm{CPU}$ 密集型已经转变为数据密集型, 而传统 OS 并没有相应的架构调整来适应这一转变.

其次, 传统操作系统不擅长为应用提供有价值的数据服务. 目前几乎所有的操作系统都把数据服 务作为上层应用. 操作系统自身只能够进行数据 (以文件或包的形式) 的标准化读写、协议数据的封 装和拆解, 但没有专门的数据栈, 不擅长处理数据中的深度信息.

此外, 传统操作系统不擅长应对新的数据相关 SLA(服务等级协议) 的要求. 例如数据采集能力方 面的类型覆盖 (数据多样性)、空间覆盖、时间连续性、数据精度等, 数据处理能力方面的实时性 (延 迟)、高效性、数据类型、数据规模等, 扩展性方面的计算性能随数据规模增加不降低、存储无上限、 扩展成本增加不超过线性、功能可扩展等, 以及能耗方面的焦耳/单位数据存储、焦耳/单位查询、平 均能耗/小时等新型度量指标.

最后, 传统操作系统不擅长满足临界数据处理请求的性能要求. 在海云计算模式下, 数据处理请 求的临界性能越来越重要, 每年春节的 12306 网站就是一个典型案例. 除此之外, 还有零负载能耗、满 负荷能耗、以指数级增速提交单一请求、迅速关掉 $80 \%$ 的节点、集中删除全部数据耗时、短时间内海 量数据查询请求、网络带宽突然降低 $50 \%$ 以上等临界情况. 但传统操作系统在这些临界情况下没有 充分的应变能力.

\subsection{2 传统操作系统的 “不作为”}

首先, 传统操作系统未能向用户直接提供数据服务. 用户仍然需要知道文件系统、磁盘目录等具 体信息, 需要了解大量的系统操作才能使用数据服务.

其次, 传统操作系统未充分利用自身对软硬件的全面感知和控制能力, 统筹考虑系统的性能、能 耗、安全的平衡. 目前操作系统虽然包含了性能优化、能耗控制、安全增强等各种功能模块, 但这些模 块之间是孤立的, 不能根据系统的运行需求进行动态平衡. 在数据挖掘和深度学习的技术下, 这种平 衡实际上是可以做到的, 而且也只能由操作系统去完成.

此外, 传统操作系统未充分利用自身的联网能力, 彼此协同形成局部智能的决策机制. 自从网络 协议栈成为操作系统的核心组件以来, 在不同计算节点上的操作系统已经相互连接, 进入网络计算时 代. 然而, 操作系统后续并没有充分利用这一联网特性, 互相交换信息, 形成局部智能的协同工作模式. 最后, 传统操作系统未记录和挖掘自身的运行历史记录数据, 为后续优化改善提供参考.

\subsection{3 传统操作系统的 “不能够”}

首先, 传统操作系统不能够为不同应用、设备和资源进行规模化的个性服务. 传统操作系统为通 用服务设计, 但随着个性化服务的需求日益增长, 其局限性愈发凸显. Android 系统等的碎片化就是一 个典型例子. 仅仅依赖于应用程序去维护数据, 无法做到全面和高效. 
其次, 传统操作系统不能够为异构节点进行按需定制和按需加载. 在不同的设备上运行时, 操作 系统应当及时获取运行节点的特征信息, 表现出动态的调节特性, 支持功能的按需定制和性能的按需 优化, 确保系统的简洁和稳定.

此外, 传统操作系统不能够实现运行状态跨设备、跨网络的连续性. 例如, 如何做到操作状态数据 和用户数据永不丢失, 每次在任意终端访问都是用户上次正在运行的环境, 这是一个普遍的需求. 目 前 Apple 利用其 AirDrop/Handoff 等在 Apple 自有设备和操作系统之间实现了这一效果. 其他的主流 操作系统仍然把这项功能交给应用层面, 导致无法实现无缝体验. 追根溯源是传统操作系统从基础结 构上并没有为跨设备、跨网络的环境而设计，而应用程序在数据的同步上又有权限等各方面的限制.

最后, 传统操作系统不能够实现自身能力随运行数据积累而不断提升. 例如, 不具备类似于生物 系统的自适应能力, 无法做到 “越用越好用”. 在网络化的条件下, 操作系统的能力 (表现为功能、性 能、能耗、安全性等各个方面) 本可以通过网络资源的共享而得到不断提升, 但传统操作系统目前的 设计架构并不支持这种自适应的升级演化能力.

综上所述, 新型计算模式的需求推动着操作系统向前发展, 数据已成为操作系统的核心要素. 以数 据为核心包括两个方面: (1) 数据作为计算的对象, 贯穿了海云协同网络计算环境的各个环节, 是信息 系统的主线, 因此数据所引发的计算需求是系统设计和运行的牵引; (2) 数据作为计算的决策依据, 需 要充分采集和挖掘, 才能为系统优化提供服务, 因此对数据的全面获取和深度处理技术是系统优化和 完善的技术驱动. 现有操作系统的结构设计和实现又无法满足对数据的直接支持. 基于对操作系统划 代的思路, 本文把数据型操作系统作为研究对象, 提出其理论和设计, 并实现了 DataOS 原型系统作为 新一代操作系统的初步探索.

文章后续的组织结构如下: 第 2 节介绍 DataOS 的理论模型, 第 3 节介绍 DataOS 的整体结构 设计, 数据栈各个层次的实现方案及验证系统的的实现方案, 第 4 节通过把 Key/Value 数据类型在 DataOS 数据栈和现有存储系统的实时性和并发性进行对比, 验证数据操作系统架构的可行性和有效 性, 第 5 节介绍学术界的相关工作, 第 6 节对全文进行总结并展望下一步工作.

\section{DataOS 的理论模型}

数据型操作系统的整体和远期设计目标是作为分布式操作系统, 以海云协同网络计算环境中的各 个单元为管理对象, 以数据为核心来提供资源管理和应用支撑, 围绕数据感知、传输、存储、处理和 服务等各个环节, 实现海云协同网络内部计算与数据资源的高度弹性化、大范围可共享和全局可管控. 为达到这一目标, 数据型操作系统的基本理念是操作系统应当能够直接管理和调度数据. 为此操作系 统需要建立与计算资源抽象类似的数据抽象. 本节将首先给出 DataOS 中引入数据抽象的理论基础, 然后给出 DataOS 中数据抽象的定义、表示和属性维护.

\section{1 数据操作系统中的数据抽象}

计算资源和数据是信息系统中主体和客体. 计算资源通常具备一些特征, 例如 $\mathrm{CPU}$ 的核数、主 频、总线频率、I/O 速率等, 从这些属性能够推导出该计算资源适合处理哪一类数据, 本文把这类属 性称为计算的数据属性. 反之, 数据也应当具备计算资源属性 (以下简称数据的计算属性), 描述其对 计算资源的需求. 在理想的计算模型中, 当计算资源的属性和数据的计算属性相匹配时, 计算资源对 数据的处理效率就会得到提升; 当系统中所有数据都被调度到与其计算属性相匹配的计算资源上处理 
时, 系统的效率达到最优. 现有操作系统通常都能获取并使用计算的数据属性, 并依此确定资源调度 算法和机制, 然而却不能有效获取并使用数据的计算属性, 进一步提升系统的效率. 将数据的计算属性 纳入调度算法, 定义计算与数据的属性匹配条件, 并实现二者的全系统内最优化匹配, 这是数据操作 系统的基本理念, 也是其克服 “不擅长”、“不作为”、“不能够”的核心技术路线.

严格起见, 我们把信息系统中有关数据抽象的概念进行形式化表述.

定义 2.1 计算的数据属性 (Data Property). 系统计算资源集合 $C=\left\{C_{1}, C_{2}, C_{3}, \ldots, C_{n}\right\}$. 对 于任意 $C_{i}$, 把有关 $C_{i}$ 的计算能力特性定义为 $C_{i}$ 的数据属性. 有关 $C_{i}$ 的数据属性的集合记为 $C_{i}$. $D P=\left\{D P_{i 1}, D P_{i 2}, \ldots, D P_{i x}\right\}$.

例 2.1 在海云协同网络环境中给定的信息系统范围内, 每一个节点就可以被认为是一个计算资 源元素. 该节点的处理器 ISA、CPU 核数、主频、多媒体编解码能力、内存大小、磁盘 I/O 速率等就 是该计算资源元素的数据属性.

定义 2.2 数据的计算属性 (Computing Property). 系统待处理的数据的集合 $D=\left\{D_{1}, D_{2}, D_{3}\right.$, $\left.\ldots, D_{m}\right\}$. 对于任意 $D_{i}$, 把有关 $D_{i}$ 某一个对计算资源的需求定义为 $D_{i}$ 的计算属性. 有关 $D_{i}$ 的计算 属性的集合记为 $D_{i} \cdot C P=\left\{C P_{i 1}, C P_{i 2}, \ldots, C P_{i x}\right\}$.

例 2.2 海云协同网络环境下, 对于一段待处理的流数据来说, 对 $\mathrm{CPU}$ 的需求、对内存的需求、 对传输带宽的需求、编解码需求、实时性需求要求等都可以成为该流数据的计算属性. 待处理数据甚 至可以将自身相关的处理代码 (可执行程序) 也作为一种计算属性.

在大多数现有操作系统中, 数据的计算属性并不是数据本身的组成部分, 只是通过上层应用的处 理过程来隐式体现, 操作系统无法直接感知, 这也是操作系统无法根据数据需求提升计算效率的根本 原因.

定义 2.3 处理效率 (Processing Efficiency) 函数. 对于某个信息系统, 其理想效率函数 $f\left(D_{i}\right)$ 定 义为系统在使用计算资源集合 $C$ 处理某个数据 $D_{i}$ 的效率. 这里的效率可以是性能、存储容量占用、 能耗等, 或者各项指标的综合, 由数据的处理目标决定. 效率函数的值越大, 代表对该项指标的满足情 况越好.

定义 2.4 属性匹配 (Property Match) 与最佳匹配 (Best Match). 对某个待处理数据 $D_{i}$, 如果系 统中存在 $C_{j}$, 对于 $\forall C P_{x} \in D_{i} . C P, \exists D P_{y} \in C_{j} . D P$, 使得 $D P_{y}$ 能够满足 $C P_{x}$, 则称 $D_{i}$ 与 $C_{j}$ 属性匹 配, 也就意味着 $D_{i}$ 可以调度到 $C_{j}$ 去处理, 记为 $M\left(D_{i}, C_{j}\right)$. 如果 $D_{i}$ 调度到 $C_{j}$ 上执行使得 $f\left(D_{i}\right)$ 的 值最大, 则称 $D_{i}$ 与 $C_{j}$ 为最佳匹配, 记为 $B M\left(D_{i}, C_{j}\right)$.

通过以上定义, 特别是数据的计算属性的引入, 系统效率最优化对于操作系统来说, 就转化为动 态规划问题, 即在处理数据集 $D=\left\{D_{1}, D_{2}, \ldots, D_{n}\right\}$ 的各个阶段, 找出最佳匹配, 使得系统总的处理效 率 $\sum_{i=1}^{n}\left(f\left(D_{i}\right)\right)$ 的值最大. 数据型操作系统的首要任务就是在操作系统层面能够引入上述数据抽象, 给出具体的定义和表示, 并且尽可能与现有的操作系统计算抽象兼容.

\subsection{DataOS 中数据的定义}

前面虽然引入了计算的数据属性和数据的计算属性等基本定义, 但还没有讨论数据在数据操作系 统中的定义. 维基百科把数据定义为可被解释为特定行为的任意符号序列, 数字化数据 (Digital Data) 则是可被计算机执行的、以电信号形式存储、记录、传输的数字、字符或符号. 在操作系统中, 数据通 常被划分为系统数据 (如系统信息、运行日志等) 和用户数据 (如文档、图片、视频等).

在 DataOS 中, 首先要界定数据的范围. 从计算系统的模型看, 目前广泛采用的冯诺伊曼体系 (von Neumann architecture) 结构中并未区分代码 (指令) 和数据, 而是将其不加区别混合存储在存储器中, 
输入给计算部件 (CPU) 时由计算部件自行判断并定义存取顺序. 自修改代码 (如加壳程序) 的存在则 进一步模糊了程序和数据的差别. 因此在 DataOS 中数据的范围不仅有传统意义上的系统数据和用户 数据, 也包含了指令和可执行程序等代码数据. 在 DataOS 的抽象模型中, 同样的对这些不同数据进 行统一抽象表示和处理, 对于它们在计算流程中的区别则由数据的计算属性来决定.

其次是数据的生命周期. 数据一旦进入 (通常通过硬件中断产生, 由设备驱动程序提取) 到操作 系统可感知和可处理的范围, DataOS 即会分配内存并以特定数据结构表示形式来描述数据, 这标志 着一个新的数据抽象的诞生. 直到为该数据抽象所分配的内存释放回收, 标志着该数据抽象消失. 生 命周期一般可以概括为产生、感知 (操作系统可操作)、传输 (包括节点内的层级移动和节点间的网络 传输)、存储、处理、服务、消亡等这样几个阶段. 中间可能有数据的拷贝、转换, 则数据的属性根据 DataOS 的规则进行相应变化.

最后是数据的属性和属性的维护. 数据的属性包括常规属性和上面提到的计算属性. 常规属性指 数据的大小、载荷、校验和等, 可以类比于一个网络包 (Packet) 的包头中所描述的各个属性. 对常规 属性的维护几乎可以沿用现有操作系统的实现, 而 DataOS 的重点在于维护数据的计算属性. 计算属 性包括数据在生命周期各个阶段的直接计算资源需求 (如 CPU、内存、存储空间、带宽、调度优先级 等需求), 以及用于推算计算资源需求的特征值. 后者并不限定范围, 没有直接标明计算需求, 但是给出 了可以推理的特征, 具有很大的弹性和灵活性, 比如数据来源 (红外传感器数据、摄像头、网页等)、数 据类型 (流式数据、Key/Value 数据)、编解码方式等, 计算单元可以从这些特征中挖掘出需要的信息, 动态确定该数据所需的计算资源.

综上所述, 我们给出 DataOS 中数据的初步定义. DataOS 中数据不仅仅是传统意义上存储信息的 单元, 更是感知、传输、处理/存储和服务等各个操作流程中的基本实体单元, 其中还包含着尽可能丰 富的关于该实体单元的计算属性. 这种包含更多信息的数据在理论上使得系统结构得到优化,特别是 对于能够掌握全局资源信息的操作系统而言.

\subsection{DataOS 中数据的表示}

人们使用操作系统以及其上的应用, 根本目标可以概括为希望获得信息. 数据作为信息的载体有 多种表现形式, 人们可以通过各种途径 (视觉、听觉、触觉等) 感知到计算机提供给的数据 (用户数 据), 并在获得这些数据的过程中, 操作系统需要加载和执行程序 (代码数据), 还会产生很多的中间数 据 (系统数据). 这些多种多样的数据外在表现形式, 都要通过在操作系统内部的数据格式来统一表示. 本小节将给出如何用操作系统内部数据格式来表示数据.

DataOS 把最基本的数据抽象称为数据单元 (Data Element). 每一个数据单元的组成如图 2 所示.

数据单元由 3 部分组成: 数据标识、数据属性和数据载荷. 其中数据标识用来唯一确定一个数据, 数据属性又分为常规属性和计算属性, 数据载荷是数据的原始部分 (对应传统操作系统中内核分配的 内存中存放、将交由应用程序处理的数据). 为了与传统操作系统的兼容, 数据标识和数据属性将在额 外的地址空间存储, 通过引用的方式与数据载荷关联.

DataOS 把若干属性相同的连续数据单元的组合称为数据块 (Data Block). 数据块包含数据块头 和连续数据单元. 其中数据块头 (Block Header) 包含了数据块的基本信息, 如数据单元的数量, 同时 包含数据单元的数据属性, 使得块内的数据单元可以共享这一属性以节省存储空间. 数据块中的数据 单元可以随时根据计算环境进行组合或分解. 在同一个数据块内的所有数据单元具有强耦合特性, 一 旦一个数据单元的属性被改变, 其他数据单元的属性或者同时跟着变化, 或者脱离当前数据块组成新 的数据块. 


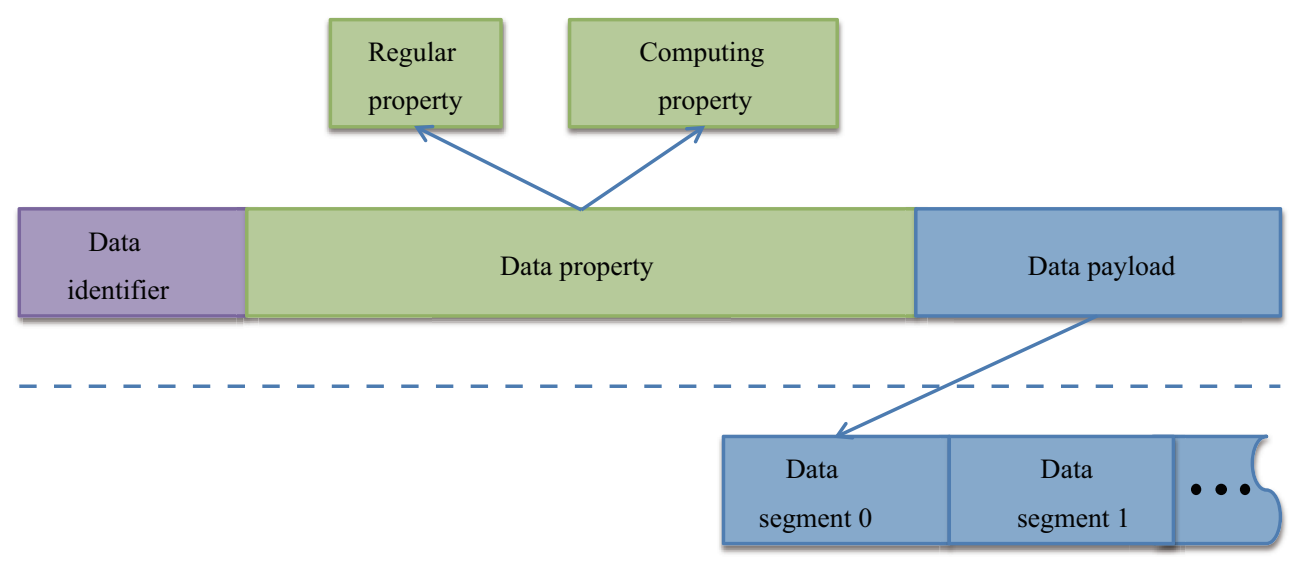

图 2 (网络版彩图) DataOS 中数据单元的结构表示

Figure 2 (Color online) The structure of a data unit in DataOS

DataOS 把若干计算属性相同, 但其他属性不一定相同的数据块的集合称为数据片 (Data Slice). 因为具有相同的计算属性, 一个数据片只需要判定一次, 即可把块中数据单元的数据载荷作为连续的 计算输入提供给计算节点. 数据片的片头 (Slice Header) 包含了数据片的基本信息, 如数据块的数量、 位置 (本地或远程) 等, 同时也包含了从数据块中挖掘出的额外信息, 例如输入的次序、速率, 以及分 解或组合的规则等. 从计算节点的角度看, 数据如果应当尽可能以数据片的形式输入, 从而提高计算 效率.

DataOS 把保存在同一个内存地址空间的数据块的集合成为一个数据分区 (Data Partition). 处于 同一个数据分区的数据块具有良好的本地性特征, 即在相互之间数据单元的移动、拷贝等操作时, 不 需要实际进行内存操作, 只需要改变指针指向即可完成. 数据分区通常代表一个节点上的数据集合, 与传统操作系统中磁盘分区的结构类似, 数据分区有分区表, 存放数据块索引.

DataOS 中一个数据分区中可以有多个数据片, 一个数据片也可以跨多个数据分区 (即来自多个 不同的节点).

需要注意的是, 以上所描述数据单元、数据块、数据片和数据分区都是操作系统的运行时概念. 节 点根据需要可以保存一份本地数据信息的持久性备份.

\subsection{DataOS 中数据的属性维护}

DataOS 中数据的产生具有多样性, 数据在节点间存在着频繁的传输、转换和组合, 数据存储和处 理节点也具有分布式特性和异构特性, 数据最终提供服务也有多种形式. 因此, 数据在海云协同网络 中除了需要有统一的抽象表示外, 还应该给出相应的属性维护规则. 数据在生命周期内通常都会发生 变化. 以最小的数据单元为例, 一个包含原始视频流的数据单元在经过 H.264 编码之后, 除有效载荷 变化之外, 数据的计算属性也会随之变化, 例如会新增编码格式的属性值, 代表期望处理节点的属性 值也变成了具有 H.264 解码器的节点. DataOS 正在维护一个关于计算属性的列表, 能够尽可能丰富 准确地涵盖各个阶段相关的计算属性. 这一列表将在后续的实践中不断补充完善或归纳合并.

为了把这一过程一般化, 我们按照数据的感知、传输、存储、处理和服务等不同流程来制定该流 程下计算属性的变化规则. 
一个数据单元在数据感知阶段, 也就是数据载荷初始赋值阶段, 需要同时对其数据的计算属性赋 值 (大部分常规属性可以从数据中直接提取). 这些计算属性包含数据的来源 (例如源自何种硬件设 备)、数据的类型 (流式、Key/Value 式等)、数据的编解码需求、数据的实时性需求、数据的持久性存 储需求 (例如是否需要写入硬盘等外存)、数据处理的可并行性、数据最终可能的服务形式等.

在完成初始赋值后, 数据如果经过传输阶段, 则会增加新的计算属性赋值, 比如传输过程的路由 选择、带宽等. 同时, 之前赋值的某些属性也要相应更新, 比如所在数据块、数据片和数据分区可能需 要重新赋值.

数据如果需要经过存储阶段, 则会增加存储介质 (例如普通硬盘、SSD 等)、存储位置、存储类 型、索引方式等新的计算属性. 同时会对其他计算属性进行更新, 例如数据是否已做持久备份、备份位 置等.

数据如果需要经过处理阶段, 则会增加处理节点、处理方式 (并行还是串行)、处理时长、处理算 法 (比如常用的分类、聚类、协同过滤等机器学习算法), 同时更新数据类型等.

数据如果需要经历服务阶段, 则会增加服务方式 (交互式、可视化、后台等)、类 SLA 的服务等级 要求、服务周期等.

需要强调的是, 从表面看 DataOS 维护这些计算属性超出了操作系统的抽象层面, 然而在分布式 环境中, 特别是复杂的海云协同网络计算环境中, 这些计算属性是决定数据迁移和资源调度的重要依 据, 也是操作系统在运行过程中持续自我完善的重要信息来源.

\section{3 数据操作系统的架构设计和原型实现}

作为数据型操作系统的首个原型系统, DataOS 的设计需求包括: (1) 实现对数据的计算属性的支 持; (2) 兼容现有的操作系统, 做到失效可回归; (3) 支持本地和分布式环境且脱离系统底层硬件的限 制. 基于此, DataOS 架构设计的整体思路是: 在现有 Linux 操作系统的基础上, 参考 $\mathrm{TCP} / \mathrm{IP}$ 网络协 议栈, 垂直整合数据相关的组件, 在 Linux 内核中增加数据栈 (Data Stack), 在兼容现有系统的同时, 实现对数据的计算属性的支持.

DataOS 的整体架构如图 3 所示.

DataOS 运行于海云协同网络的计算节点, 复用 Linux 已有的设备驱动、网络协议栈、文件系统 等作为硬件抽象层, 在此之上构建包含数据感知、数据传输、数据存储/处理、数据服务的数据栈. 数 据栈运行在内核态, 数据从应用程序输入数据栈后经过各个模块的处理, 最后从数据栈输出给应用程 序. 数据栈参考了 ISO/OSI 的 7 层网络协议栈模型, 希望把在单机和在分布式环境下有关数据的共性 操作提炼出来, 支持数据的计算属性, 为应用提供通用的数据支撑, 为数据型操作系统探索一种可实 现的技术架构范式.

下面将对数据栈每一个层次的功能设计进行详细介绍.

\section{1 数据感知层}

数据感知层负责在本地或网络的数据到来之后, 将其创建为 DataOS 后续数据栈可以理解的数据 单元格式.

由于数据的来源多种多样, 数据感知层引入数据设备 (Data Device) 的概念, 用来抽象本地设备、 本地文件系统、操作系统自身、外部节点等所有可能的数据源. 新的数据源可以注册为数据设备, 从 


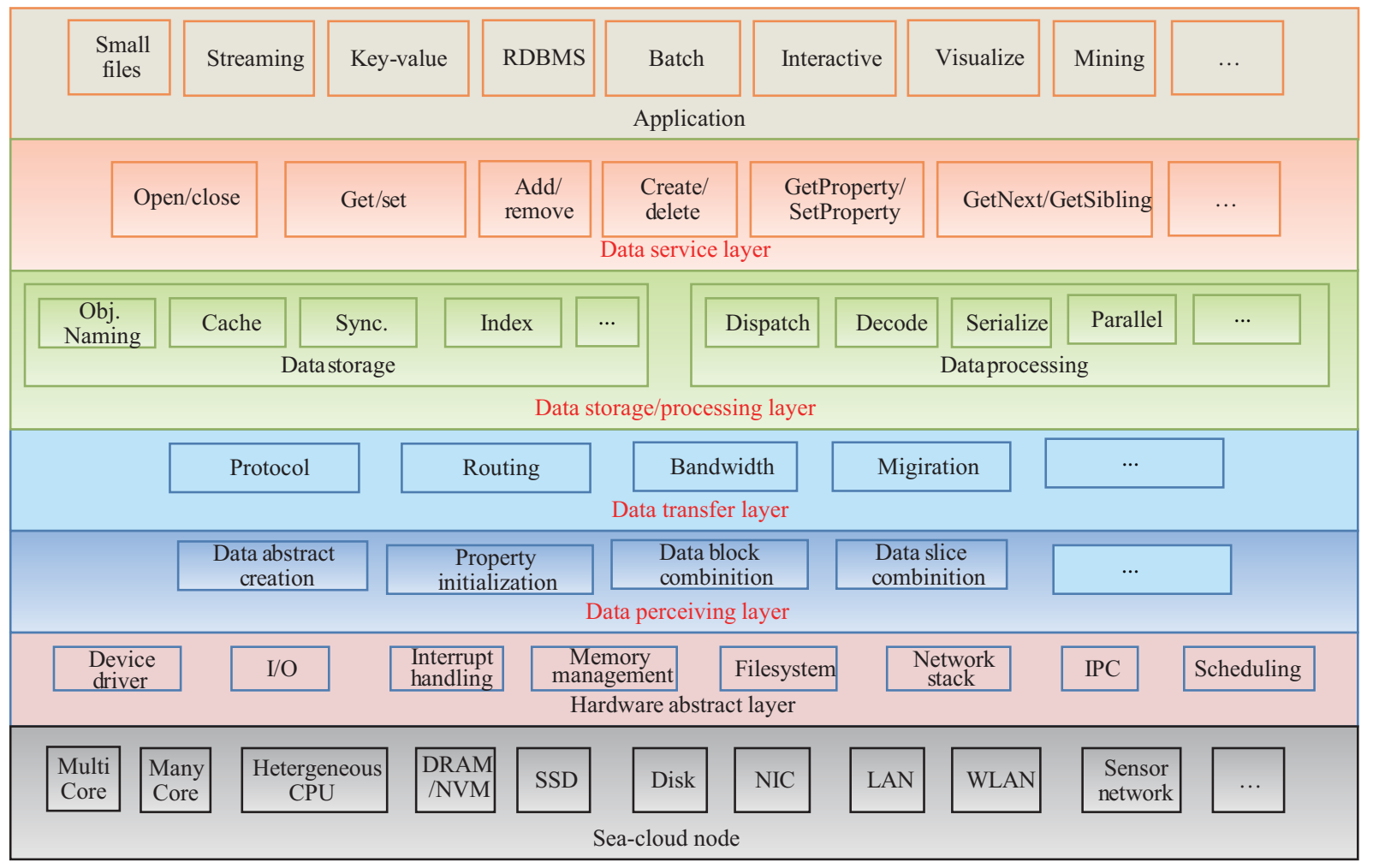

图 3 (网络版彩图) DataOS 整体架构图

Figure 3 (Color online) The architecture of DataOS

而使其数据纳入数据感知层的处理流程.

通过 Data Device 注册时提供的信息, 感知层可以获得关于数据单元的数据来源、类型等绝大部 分属性的初始值, 然后结合当前的计算环境特征, 完成对数据单元其他计算属性的初始赋值. 同时, 感 知层会把新产生的数据单元链入相应的数据块和数据片, 为批量数据操作和批量数据调度奠定基础.

\section{2 数据传输层}

数据传输层主要解决数据在节点内部和节点间的流动问题. 数据单元在创建之后往往要么在本节 点进行存储处理, 要么向上层应用传递 (对应于传统操作系统中向用户态拷贝的操作), 要么向其他节 点进行传输. 在 DataOS 的应用场景中, 前两种情况意味着数据的生命周期将只局限于本地节点, 传输 层只需要对数据的属性进行赋值, 然后交由数据栈的下一层次处理即可. 对于最后一种情况, 即数据在 节点间迁移调度的情况, 则需要获取数据的传输协议、可能的路由选择方式、期望的传输带宽以及相 应的传输策略. 其中传输策略是综合数据单元的计算属性后作出的启发式判断, 是实现海云协同网络 “减少数据传输、就近处理” 原则的重要基础.

为了方便 DataOS 对计算属性的更新和赋值, 传输层引入了数据总线 (Data Bus) 的概念. 数据总 线为常用的数据传输协议, 如 $\mathrm{TCP} / \mathrm{IP}$ 或者更加上层的 $\mathrm{HTTP}$ 协议、基于广播方式的蓝牙等, 提供注 册接口. 协议可以在注册到数据总线时, 提供尽可能多的信息. 当数据单元在传输层与某一种注册协 议关联时, 有关其传输过程的计算属性就可以从协议中获取. 


\section{3 数据存储/处理层}

之所以把数据存储和处理放在同一层次, 是因为二者通常是交叉的. 这一层次的主要目的是把现 有主流的分布式数据存储引擎以及主流的分布式数据处理引擎的共性技术提取出来, 放入内核层次, 提升系统效率. 这样做的原因在本文下一节将有详细的验证.

在数据存储部分, 将纳入现有关系型、NoSQL 型数据库的一些底层机制, 如对象命名、缓存、数 据同步、数据索引等, 并提供注册机制. 对于原来实现在用户态的数据存储软件, 如存储 Key/Value 型 数据的 Memcached, 需要对其核心部分在操作系统的内核态中实现, 并注册到数据存储部分, 成为一 类存储引擎实例. 在数据单元进入存储层次后, 将根据不同的存储方式调度到相应的存储引擎, 并更 新相应的数据属性.

数据处理部分与存储部分类似, 将对现有的批量数据处理、迭代数据处理、流式数据处理的共性 部分提取出来纳入操作系统内核态, 对典型的用户态数据处理软件, 以数据处理引擎的方式在内核态 中实现并注册. 数据存储/处理层的难点在于分布式环境中的节点间协同. 为此 $\mathrm{DataOS}$ 在这一次层专 门提供了远程访问接口, 作为节点间数据的直接通道.

\section{4 数据服务层}

数据服务层有两个核心功能: (1) 提供数据服务接口, 为上层应用中的各种数据需求提供高效服 务; (2) 根据数据属性, 特别是计算属性, 引入数据挖掘算法, 为系统自身和海云协同网络环境中的其 他关联节点 (如同一集群) 的优化提供服务.

DataOS 总结了 Key/Value 型数据、流数据 (包括时空流数据)、图数据等不同数据类型的操作接 口, 给出了一个数据服务层的接口集合. 作为第一步, 这些接口目前大量借用了 Linux 内核中现有的 socket 接口和文件系统接口. 后续实践中将对这一集合进行持续完善和优化.

对于计算属性的挖掘, 目前 DataOS 希望提供挖掘和学习算法的注册机制. 一个注册算法可以遍 历系统中的数据抽象的所有属性, 作为算法的输入, 之后能够输出有价值的系统优化策略, 从而实现 数据的 “取之于操作系统, 用之于操作系统”.

\subsection{DataOS 概念验证原型的实现}

作为 DataOS 的第 1 步, 我们首先实现一个能够在架构层面进行验证的原型系统, 即基于数据栈 的思路和架构, 对现有操作系统进行改进, 实现对典型数据业务的支持且达到更好的效果, 初步证明 通过数据栈向操作系统引入数据抽象的有效性.

\subsection{1 验证背景}

这里我们选择了互联网在线事务处理型 (OLTP) 任务作为典型业务, 其对高并发、低延迟、高可 扩展的数据存储都有需求, 但现有操作系统和之上的 NoSQL 系统并不能很好支持. 文献 [2] 对现有主 流系统进行了一系列评测, 发现当并发访问量增大时, 单个请求的响应时间有增加的趋势; 即使并发 访问量不高, 当系统中的总数据量累积到一定程度时, 单个请求的响应时间也会显著增加; 同时, 当增 加节点数时, 所能处理的并发请求数并不能线性增加. 通过对主流操作系统和 NoSQL 系统进行分析, 本文认为这些系统存在以下几个问题. 
(1) 仍然采用传统多线程模型, 对多核 CPU 的利用率不高. 首先, 没有对多线程进行有效管理, 使 得多核 $\mathrm{CPU}$ 负载不均衡, 其次, 请求在处理时会独占线程, 线程时常会阻塞在 $\mathrm{I} / \mathrm{O}$ 上, 其他请求难以 得到公平处理.

(2) 仍然采用传统的索引结构, 不支持并发访问. 为了在多线程并发访问时保证索引结构的一致 性, 多数系统都采用锁对索引进行保护, 由于锁是操作系统内核提供的机制, 因此在用户态访问锁时 会涉及进程的上下文切换, 当处理海量请求时, 频繁地加锁和解锁会显著地降低系统的性能.

(3) 对存储空间的管理不高效, 没有对数据进行有效分类并安排数据在内存和磁盘上的分布, 如 LevelDB 对新数据提供较好的读写性能, 但对旧数据访问的性能较差, 又如 MongoDB 的内存浪费严 重, 频繁随机访问时, 会因内存不足导致页面频繁交换, 性能降低.

(4) 多数系统的访问接口基于 Socket 实现. 服务器端在输入输出时, 需要频繁地进行上下文切换, 同时还涉及用户态一内核态之间的重复数据拷贝, 增加了通信的延迟.

(5) 多数系统采用集中式的元数据管理方式, 制约了集群的扩展能力.

对以上因素一言以蔽之, 问题的根源之一在于操作系统与运行于用户态的数据管理系统缺乏有效 配合. 换言之, 操作系统没有能够围绕数据发挥主导作用, 这也是 DataOS 之所以希望在内核态中实现 数据栈的原因.

\subsection{2 验证原型的实现}

为解决以上问题, DataOS 选择典型的 Key/Value 类型存储作为验证对象, 对比当前流行的 Key/ Value 存储软件 Memcached, 在内核态数据栈框架下实现了相似的 Key/Value 数据存储机制, 作为存 储引擎注册到数据存储层, 同时在数据服务层增加相应的访问接口. 验证系统的架构如图 4 所示, 主 要涉及访问接口和存储引擎两部分.

DataOS 提供了两种访问接口: 基于 Socket 的通用访问接口; 基于 VFS 的本地访问接口. 远程访 问接口基于内核态 Socket 实现. 存储节点开启内核态 Socket 监听来自 Client 的连接, 当接收到连接 后, 交由请求处理层的工作线程, 之后的数据传输和处理均由工作线程完成. 为了提高效率, 存储节点 并不会以阻塞的形式监听新的请求, 而是采用异步 I/O 的方式. DataOS 在内核中为监听 Socket 注册 了 sk_state_change 等回调函数, 在其中实现了接收和分发连接的逻辑, 当 Socket 上有数据到达或者状 态发生改变时, 其回调函数会被中断处理例程调用, 从而自动执行接收和分发新连接的逻辑, 同时实现 对数据属性的更新和赋值. 针对本地访问, DataOS 以系统调用的形式提供接口给客户端. 为了保证可 移植性和部署的便利性, DataOS 没有在内核中增加新的系统调用, 而是复用内核中已有的文件系统 接口, 在内核中实现了接收和分发请求的逻辑, 请求的处理则交由工作线程完成. 在 Socket 接口和文 件系统接口的基础上, DataOS 在数据服务层封装了 5 种常用的 Key/Value 操作函数 (Get, Set, Add, Replace, Delete).

在内核内部, DataOS 实现了 Key/Value 的存储引擎, 并注册到数据存储层. 每种 Key/Value 操作 都对应一种 Key/Value 请求, 存储引擎按协议接收相应的 Key/Value 请求, 完成与 Memcached 类似 的内部存储处理机制 (这里不再赘述), 之后提取出结果并返回给请求者, 期间涉及的同步互斥、异常 等处理都在存储引擎内部处理, 对客户端透明. 


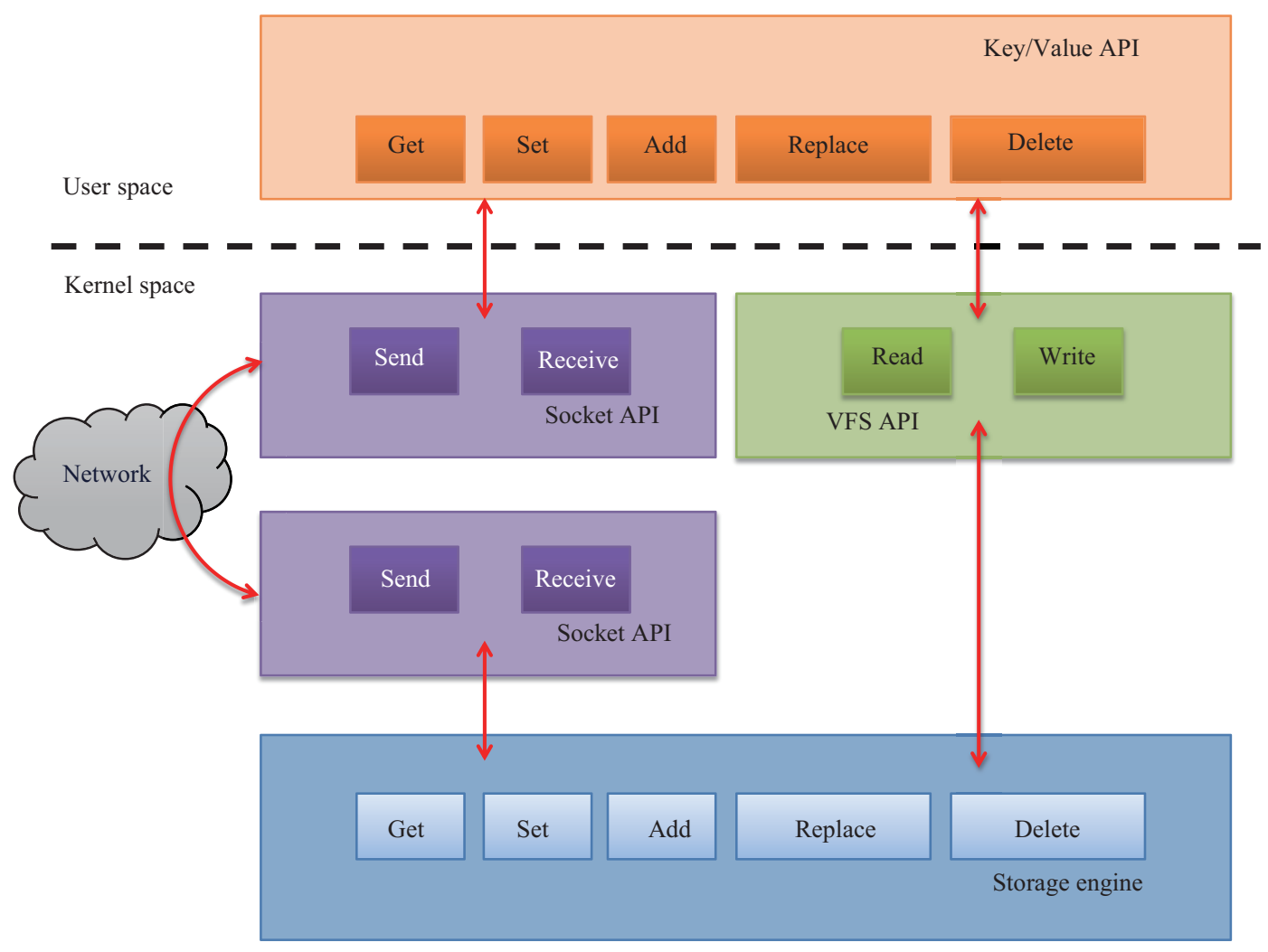

图 4 (网络版彩图) 针对 Key/Value 数据的 DataOS 数据存储层和数据服务层的设计

Figure 4 (Color online) The DataOS storage layer and service layer for Key/Value storage

\section{4 实验设计及结果}

本节以 Memcached 为对比对象, 设计了两组实验对 DataOS 原型系统的实时性、并发性进行测 评. 实时性测试的目的是考察 DataOS 处理请求的速度, 测试其响应时间与主流 Key/Value 存储系统 的区别; 并发性测试的目的是考察 DataOS 处理请求的并发度, 测试单位时间内能够处理多少并发请 求. 为了单纯对比架构方面的优势, 在实验过程中, DataOS 对内存中输入和输出数据进行了抽象封 装, 但并不对数据属性进行空间分配和赋值操作. 同时作为对原型系统的验证, 本文实验在单节点上进 行, 配置如下: 24 核 CPU, $64 \mathrm{G}$ 内存, $10 \mathrm{~T}$ 硬盘, 操作系统分别采用未修改过的 Ubuntu Server 12.04 (Linux Kernel 版本 3.8.13), 以及基于此系统修改后得到的 DataOS 原型. 另外还有一台物理机作为客 户端, 模拟海量并发请求, 通过千兆网络与 Memcached 节点和 DataOS 节点互联.

\section{1 实时性}

本实验考察 DataOS 处理请求的速度, 测试其响应时间是否低于主流 Key/Value 存储系统. 为了 避免缓存的干扰, 本实验数据总量不超过 DataOS 节点的内存缓存大小, 从而保证数据全部在内存中 存取. 本实验首先利用单个客户端, 向 DataOS 节点连续发起一批写请求 (Set), 等待响应, 统计响应 时间, 然后逐渐增加每批请求中的请求数量, 统计响应时间在请求数量逐渐增大时的变化情况. 之后 以 Memcached 作为对比对象, 采用相同的实验过程对其进行测试. 

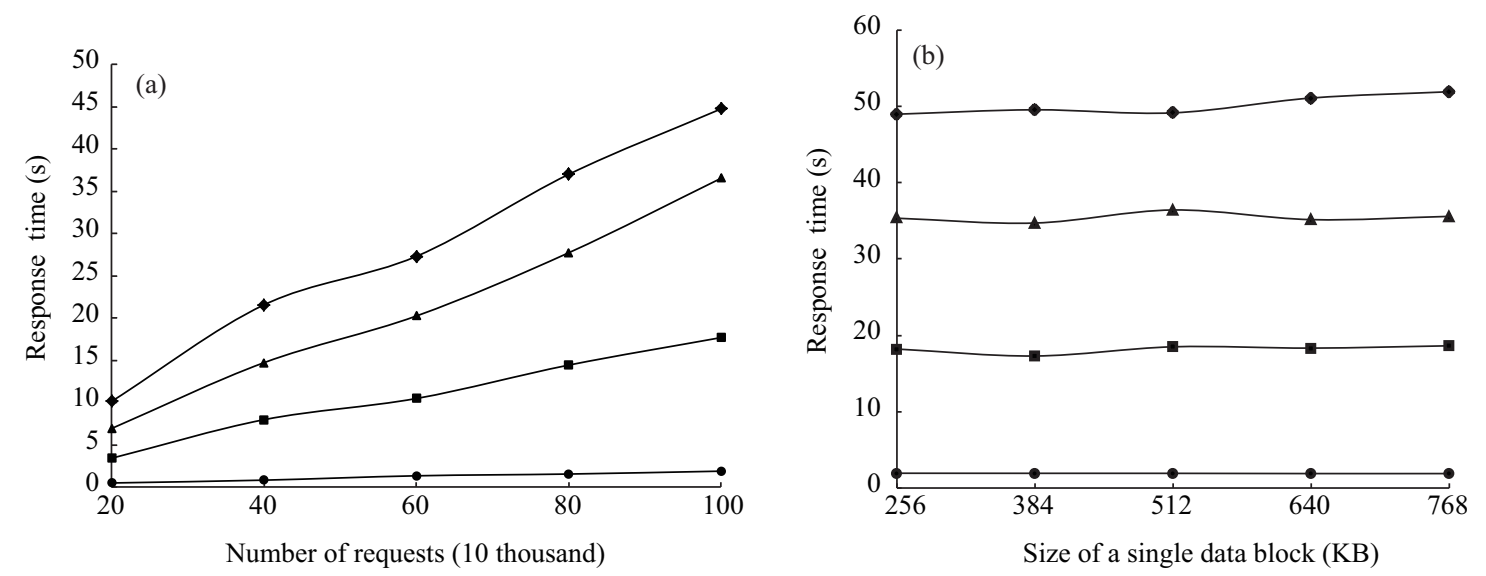

$\rightarrow$ DataOS-Local $\rightarrow$ DataOS-TCP

Memcached-Local

$\rightarrow$ Memcached-TCP

图 5 基于不同请求数的实时性对比测试和基于数据量的实时性对比测试

Figure 5 (a) The result of request-number based real-time experiment; (b) the result of data-amount based real-time experiment

由于 DataOS 和 Memcached 都有两种访问接口, DataOS 提供基于文件系统的接口 (DataOSLocal)、基于 TCP Socket 的接口 (DataOS-TCP), Memcached 提供了基于 Unix Socket 的接口 (Memcached-Local)、基于 TCP Socket 的接口 (Memcached-TCP). 为了保证测试的全面性, 本实验分别对以 上 4 种接口进行测试. 图 5(a) 为实验结果, 记录的是随着客户端发起的请求数量的增加, 服务器端处 理这些请求所需时间 (即响应时间) 的变化.

从结果可以看出, DataOS 的响应时间显著低于 Memcached, 其中, DataOS-Local 的响应时间最 低, 即使在处理 100 万个请求时, 只消耗了约 $2 \mathrm{~s}$ 的时间. 之所以有如此大的差距, 主要是由于 DataOS 存储引擎运行在内核态, 客户端可以直接访问 DataOS 中的数据, 相比需要经过内核态 一 用户态切 换、IPC 机制才能访问的 Memcached, 数据拷贝以及状态切换的次数更少. 基于同样的原因, 在 TCP 接口下 DataOS 响应时间也明显比 Memcached 低, 与预期的效果一致.

为了进一步探究导致性能差距的原因, 本文进行了另一场实验, 考察数据拷贝的数据量对性能产 生的影响. 本实验首先利用单个客户端, 向 DataOS 节点发起大量写请求 (Set), 等待响应, 统计响应 时间, 然后保持请求数不变, 而逐渐增加单个请求中数据的大小, 统计响应时间在数据量逐渐增大时 的变化情况. 之后以 Memcached 作为对比对象, 采用相同的实验过程对其进行测试. 与上一个实验类 似, 本实验分别对 DataOS/Memcached 的 4 种接口进行测试. 图 5(b) 为实验结果, 其中横轴表示单个 Key/Value 数据的大小, 纵轴表示响应时间.

从结果可以看出, 无论是 DataOS 还是 Memcached, 当请求的数据量逐渐增大时, 其响应时间并 不会明显增加. 考虑到实验过程中保持请求数不变, 但增加了每个请求中的数据大小, 即实验过程中 涉及的拷贝数据量是逐渐增大的, 因此, 可以认为数据楛贝不是影响响应时间的关键因素. 回顾上一个 实验, 其保持每个请求中数据大小不变, 而增加请求的数量, 结果导致响应时间增加. 增加请求数量意 味着状态切换次数的增多, 因此, 可以认为状态切换才是影响响应时间的关键因素. 在运行过程中, 位 于内核态的 DataOS 存储引擎相比用户态的 Memcached, 将产生更少的状态切换, 因此性能更好. 实 验结果证明了基于内核的存储系统的有效性. 

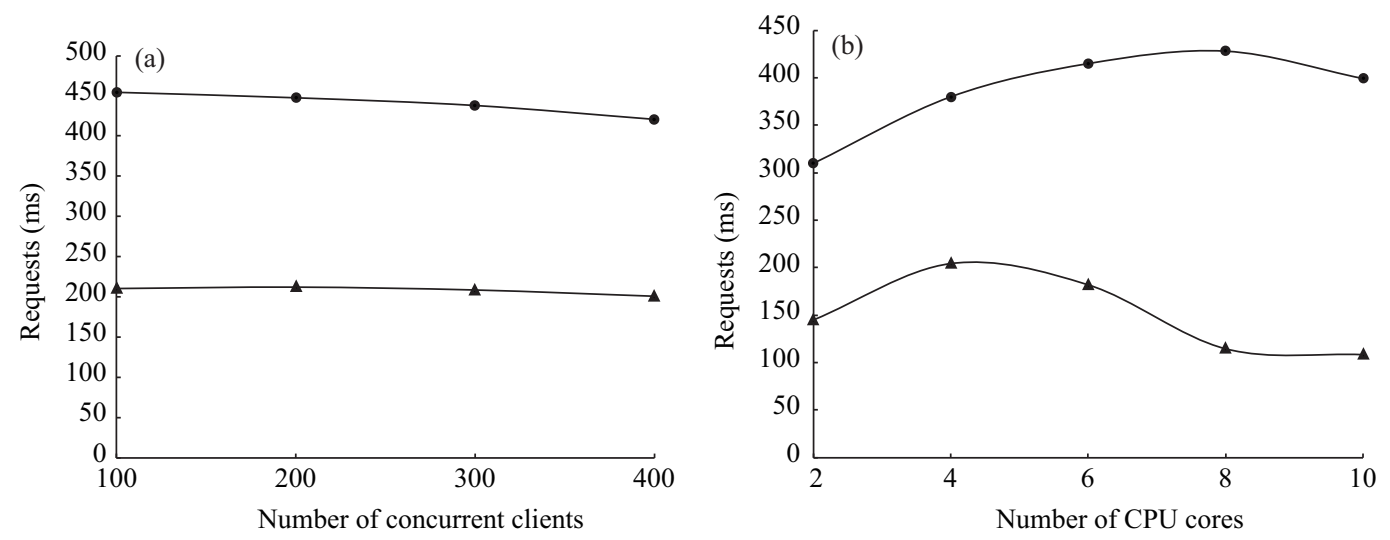

图 6 基于并发客户端数的并发性测试和基于 CPU 核数的并发性测试

Figure 6 (a) The result of client-number based concurrency experiment; (b) the result of core-number based concurrency experiment

\section{2 并发性}

本实验考察 DataOS 处理请求的并发度, 测试单位时间内能够处理多少并发请求. 实验利用多个 客户端, 并发地向 DataOS 节点发起写请求 (Set), 统计单位时间内 DataOS 所能够处理的并发事务数 (TPS), 并逐渐增加客户端的数量, 以统计 TPS 的变化趋势. 然后以 Memcached 作为对比对象, 采用 相同的实验过程对其进行测试. 为了保证测试的公平性, 本实验通过参数调整 DataOS 和 Memcached 的内置线程数, 使其能够利用等量的多核 CPU 并发处理请求.

图 6(a) 为实验结果, 展示了随着客户端数量的增多, DataOS 和 Memcached 的 TPS 的变化. 其 中横轴为客户端 (Memslap) 的数量, 纵轴为 DataOS/Memcached 的每毫秒所处理的并发请求数.

从结果可以看出, DataOS 的 TPS 始终高于 Memcached, 保持近 2 倍的优势. 随着客户端数量的 增多, DataOS 和 Memcached 的 TPS 都有下降的趋势, 这是由于 DataOS/Memcached 的性能已经处 于饱和状态 (CPU 使用率 100\%), 随着客户端的增多, 内部线程的竞争更加激烈, 导致并发性下降.

为了进一步探究 CPU 核数对 DataOS 和 Memcached 并发性的影响, 本文进行了另一场实验. 利 用多个客户端, 并发地向 DataOS 节点发起大量写请求 (Set), 统计单位时间内 DataOS 所能够处理的 并发事务数 (TPS), 然后逐渐增加 DataOS 内置线程数 (等同于增加 CPU 核数), 统计 TPS 的变化趋 势. 最后以 Memcached 作为对比对象, 采用相同的实验过程对其进行测试.

图 6(b) 为实验结果, 展示了随着 $\mathrm{CPU}$ 核数的增加, DataOS 和 Memcached 的 TPS 的变化, 其中 横轴为 CPU 核数, 纵轴为 DataOS/Memcached 的每毫秒所处理的并发请求数.

从结果可以看出, 随着 CPU 核数的增加, DataOS 的 TPS 平稳增加, 具有很好的并发性和可扩展 性, 而 Memcached 的波动较大, 出现了 4 线程 Memcached 的 TPS 超过了 8 线程 Memcached 的情况, 这也印证了 Memcached 在设计时针对 4 线程进行了优化 ${ }^{[2]}$. 无论何种情况下, DataOS 的 TPS 始终 高于 Memcached, 这是由于 DataOS 存储引擎在内核态, 能够利用内核多线程充分挖掘多核 CPU 的 并发性. 实验结果表明 DataOS 在并发处理机制方面具有明显优势.

以上实验仅从系统架构设计的角度, 验证 DataOS 及其数据栈是可行和有效的, 还没有涉及数据 属性的相关操作, 以及基于数据属性的全系统优化. 后续的验证工作将随 DataOS 的开发同步展开, 逐 
步面向更加复杂、更加真实的海云协同网络计算环境, 并引入数据挖掘算法, 真正体现在 DataOS 架 构下基于数据的智能化演进的优势.

\section{5 相关工作}

数据操作系统希望以新的操作系统架构来适应数据为核心的计算模式需求, 既涉及操作系统领域, 也涉及数据管理和大数据相关的领域.

关于研发一个不同于主流操作系统的新操作系统需要注意的事项, IBM 历时 10 年 (1996 2006) 的 K42 内核研究项目 ${ }^{[3]}$ 做了非常全面的总结 ${ }^{[4]}$, 提出了操作系统的设计要满足可定制 (Customizable)、 可伸缩 (Scalable)、可维护 (Maintainable) 以及可兼容 (Compatible) 的要求, 为 DataOS 提供了架构设 计的参考. DataOS 希望未来能发展为一个通用型的操作系统, 因此在设计之初就考虑重用 Linux 这 样定制性、伸缩性、可维护性已经非常成熟的操作系统, 且与之保持兼容. 以 $\mathrm{Xen}^{[5]}$ 为代表的虚拟化, 以及 Minix ${ }^{[6]}$ 和 L4 ${ }^{[7]}$ 为代表的微内核从学术界出现到工业界崛起, 表明新的操作系统只要有其独 到之处, 就会有生命力.

近年来, 多核和分布式操作系统受到进一步的关注. 学术界对操作系统的多核支持情况进行了反 思 ${ }^{[8]}$, 提出了新的操作系统设计架构. 一般来说, 操作系统对分布式环境的支持可以从两个方面入手, 一种是自上而下, 在用户态通过应用程序和网络协议来实现, 现有的大多数集群系统都可以认为是这 种技术方案; 另一种是自下而上, 在硬件和操作系统之间加入消息机制, 支持多内核 (Multi-Kernel) 的 运行方式来实现. 苏黎世理工学院的 BarrelFish ${ }^{[9,10]}$ 是后者的典型代表, 能在不同的 CPU 核上运行 多个操作系统内核, 并在此基础上实现了能够将 CPU 核和内核代码动态解耦合的机制 ${ }^{[11]}$, 而多核上 的这些成果很容易扩展到分布式系统中. 这两种方法中前者只是针对特定应用的分布式, 后者则可能 存在应用兼容性的问题. 作为同样面向分布式系统的 DataOS 采取了第 3 种办法, 将分布式特性纳入 内核态作为内核的标准组件, 同时基于数据实现分布式协同中节点的协作.

在操作系统的架构改进和数据抽象方面, 还有一些最新的研究成果. Arrakis ${ }^{[12]}$ 允许应用程序直 接访问虚拟化后的 I/O 设备,而不需要内核仲裁, 使得 NoSQL 存储的吞吐率提升了 9 倍, 这也充分证 明了现有 NoSQL 大数据存储系统与操作系统没有很好配合, 有很大的提升空间. Jitk ${ }^{[13]}$ 在内核里实 现了一个可信解释器, 为内核动态增加功能和指定策略提供了机制, 这也是未来 DataOS 在内核中使 用数据属性调整运行策略的可选方案. 与 DataOS 数据栈架构的优势类似, IX 项目 ${ }^{[14]}$ 通过内核中的 数据层 (Data-Plane) 实现了网络传输的高吞吐低延迟. Pebble 项目 ${ }^{[15]}$ 把应用程序的数据管理机制 进行了归纳后, 提出了应用层数据对象的抽象概念, 能够表达 email、文档、银行账户、笔记记录等不 同的对象, 并得出结论在现代操作系统中嵌入高层的存储抽象是可行的.

在面向大数据方面也出现了一些新型的分布式操作系统. Berkeley AMP 实验室针对数据中心环 境在 2011 年提出了 Mesos ${ }^{[16]}$. Mesos 主要实现对集群中 3 个 “角色” 的组织协调和有序执行: 控制节 点, 工作节点, 计算框架. 控制节点一方面获取计算框架的资源需求; 另一方面获取各个工作节点的可 用资源情况, 并根据调度策略将工作节点的资源分配给计算框架执行作业. 围绕 Mesos, Berkeley 提出 了一个数据分析处理平台 BDAS, 其包括内存中并行处理的框架 Spark, 以及数据查询引擎 SparkSQL, 基于分布式系统的机器学习系统 MLlib、可靠的分布式图计算框架 GraphX 等. 这些围绕大数据的工 作提出了一系列数据传输、存储和处理的需求, 也总结了如何利用数据的特点 (即本文中所提出的数 据属性) 来优化分布式计算.

面向海云协同网络环境, 普林斯顿大学的 Arial 等研究了将在广域范围内所采集到的数据存储在 
终端的可行性, 同时设计了 JetStream ${ }^{[17]}$ 系统, 实现根据网络状况及数据质量等动态调整信息服务的 数据传输方式以及数据质量. IBM 研究院及微软研究院针对家庭中联网的设备, 如可远程控制的锁、 灯具、摄像头、动作传感器等, 实现了 $\mathrm{HomeOS}^{[18]}$ 平台, 将整个家庭的各类计算设备当做一台计算机 来进行设计; 通过抽象的应用程序接口, 可在不同设备中分配任务, 并为用户提供一个家庭环境的管理 界面, 该系统已经在美国真实家庭中进行了部署和测试. 基于 HomeOS 所提供的 API, 第三方可以较 容易地开发应用程序. 这些都从不同角度描绘了 DataOS 可能应用场景.

\section{6 结论和未来工作}

以数据为核心是新型计算模式日益显现的一个重要特征, 本文从操作系统的角度, 提出了一种新 型操作系统——数据操作系统, 核心是在操作系统中引入数据的计算属性, 叙述了其第一个概念验证 原型 DataOS 的架构设计和数据栈的具体实现方案, 并通过对 Key/Value 数据类型的实验, 验证其可 行性和有效性. 从操作系统的发展历史看, 最早始于 BSD 操作系统的网络协议栈的引入, 使得计算技 术进入了计算互联的互联网时代. DataOS 中引入数据栈作为操作系统的核心功能部件, 希望能够为 操作系统提供智能化的基础, 即基于数据来解决操作系统 “不擅长”、“不作为”、“不能够” 的问题, 从 而有望开启数据互联的新时代.

数据操作系统的构建和完善还有很多工作要做. 仅从设计层面上看, 就有安全性、模块化、可伸 缩性等诸多问题需要深入考虑. 下一步我们将在数据栈中支持更多的数据类型, 并结合网络协议栈将 数据栈扩展到分布式环境中, 同时引入数据挖掘技术实现系统的智能调优, 在不断实践的过程中完善 DataOS 的理论和架构, 最终希望数据栈能够像网络协议栈一样, 成为新一代操作系统的设计范式和 核心部件, 比现有操作系统更好地满足海云计算环境的需求.

\section{参考文献}

1 Sun N H, Xu Z W, Li G J. Sea computing: the new calculation model of the internet of things. Commun China Comput Fed, 2010, 2: 39-43 [孙凝军, 徐志伟, 李国杰. 海计算: 物联网的新型计算模型. 中国计算机学会通讯, 2010, 2: $39-43]$

2 Gandini A, Gribaudo M, Knottenbelt W J, et al. Performance Evaluation of NoSQL Databases. Berlin: Springer, 2014. $16-29$

3 Krieger O, Auslander M, Rosenburg B, et al. K42: building a complete operating system. SIGOPS Oper Syst Rev, 2006, 40: 133-145

4 Wisniewski R W, Da Silva D, Auslander M, et al. K42: lessons for the OS community. SIGOPS Oper Syst Rev, 2008, 42: $5-12$

5 Barham P, Dragovic B, Fraser K, et al. Xen and the art of virtualization. SIGOPS Oper Syst Rev, 2003, 37: 164-177

6 Herder J N, Bos H, Gras B, et al. Minix 3: a highly reliable, self-repairing operating system. SIGOPS Oper Syst Rev, 2006, 40: 80-89

7 Heiser G, Elphinstone K, Kuz I, et al. Towards trustworthy computing systems: taking microkernels to the next level. SIGOPS Oper Syst Rev, 2007, 41: 3-11

8 Baumann A, Peter S, Schüpbach A, et al. Your computer is already a distributed system. Why isn't your OS? HotOS, 2009

9 Baumann A, Barham P, Dagand P E, et al. The multikernel: a new os architecture for scalable multicore systems. In: Proceedings of the ACM SIGOPS 22nd Symposium on Operating Systems Principles, New York, 2009. 29-44

10 Holland D A, Seltzer M I. Multicore OSes: looking forward from 1991, er, 2011. In: Proceedings of the 13th USENIX Conference on Hot Topics in Operating Systems, Berkeley, 2011. 33 
11 Zellweger G, Gerber S, Kourtis K, et al. Decoupling cores, kernels, and operating systems. In: Proceedings of 11th USENIX Symposium on Operating Systems Design and Implementation (OSDI 14), Broomfield, 2014. 17-31

12 Peter S, Li J, Zhang I, et al. Arrakis: the operating system is the control plane. In: Proceedings of 11th USENIX Symposium on Operating Systems Design and Implementation (OSDI 14), Broomfield, 2014. 1-16

13 Wang X, Lazar D, Zeldovich N, et al. Jitk: a trustworthy in-kernel interpreter infrastructure. In: Proceedings of 11th USENIX Symposium on Operating Systems Design and Implementation (OSDI 14), Broomfield, 2014. 33-47

14 Belay A, Prekas G, Klimovic A, et al. IX: a protected dataplane operating system for high throughput and low latency. In: Proceedings of 11th USENIX Symposium on Operating Systems Design and Implementation (OSDI 14), Broomfield, 2014. 49-65

15 Spahn R, Bell J, Lee M, et al. Pebbles: fine-grained data management abstractions for modern operating systems. In: Proceedings of 11th USENIX Symposium on Operating Systems Design and Implementation (OSDI 14), Broomfield, 2014. $113-129$

16 Hindman B, Konwinski A, Zaharia M, et al. Mesos: a platform for fine-grained resource sharing in the data center. In: Proceedings of the 8th USENIX Conference on Networked Systems Design and Implementation, Berkeley, 2011. 295-308

17 Rabkin A, Arye M, Sen S, et al. Making every bit count in wide-area analytics. In: Proceedings of the 14th Workshop on Hot Topics in Operating Systems, Berkeley, 2013

18 Dixon C, Mahajan R, Agarwal S, et al. An operating system for the home. In: Proceedings of the 9th USENIX Symposium on Networked Systems Design and Implementation (NSDI 12), San Jose, 2012. 337-352

\title{
DataOS: principle, design and prototype implementation
}

\author{
LI MingShu ${ }^{1,2}$, ZHAO Chen $^{1,2}$, WU YanJun ${ }^{1,2 *} \&$ XIE PeiDong ${ }^{1}$ \\ 1 Institute of Software, Chinese Academy of Sciences, Beijing 100190, China; \\ 2 State Key Lab of Computer Science, Beijing 100190, China \\ *E-mail: yanjun@iscas.ac.cn
}

\begin{abstract}
The modern operating system (OS) is inclined to be less dependent on devices and more involved with data. Data manipulation now tends to be the core functionality of future OSes. This is also one principle of the sea-cloud computing environment. By analyzing the challenges and problems of traditional OSes, this paper proposes the design and implementation paradigm of a next-generation OS, DataOS. DataOS introduces data abstraction and an in-kernel data stack that can directly support data collection, transfer, processing, storing, and service-providing. DataOS presents a novel solution not only for performance improvement of current big data systems but also for operating system evolvement. As a proof of concept, we implement the first version of the DataOS prototype that includes a data stack and ports the Key/Value storage system. The results of experiments confirm that DataOS outperforms the classical Memcached in terms of both real-time and concurrency and validate the feasibility and efficiency of the philosophy and design of DataOS.
\end{abstract}

Keywords operating system, data operating system, data stack, big data, distributed system 


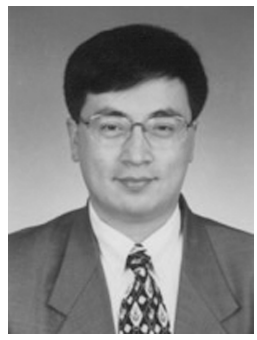

LI MingShu was born in 1966. He received a Ph.D. degree from the Department of Computer Science, Harbin Institute of Technology in 1993. Currently, he is a research professor at the Institute of Software, Chinese Academy of Sciences. His research interests include the deep design of operating systems (including secure operating systems and data operating systems), trustworthy software processes, and fundamental software and hardware technologies and applications. He is the deputy chief engineer of "Core Electronic Devices, High-end Generic Chips and Fundamental Software" of the National Science and Technology Major Project. He is a fellow of the China Computer Federation (CCF).

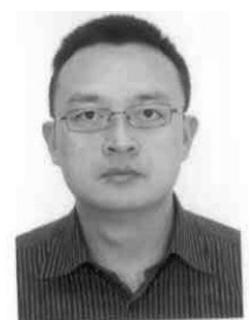

WU YanJun was born in 1979. $\mathrm{He}$ received a Ph.D. degree from the Institute of Software, Chinese Academy of Sciences (ISCAS). Currently he is a research professor at ISCAS and his research interests include operating systems and system security.

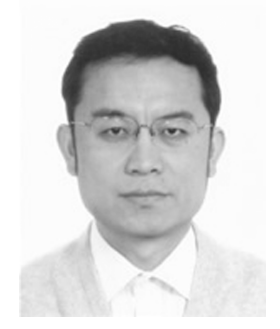

ZHAO Chen was born in 1967. He received a Ph.D. degree from the Institute of Software, Chinese Academy of Sciences in 2000 (ISCAS). Currently, he is a research professor at ISCAS. His research interests include compiling, auto-testing, and operating system and networking software. He is the deputy director and chief engineer of ISCAS. 